\title{
Wide-field X-ray Monitor on HETE-2
}

\author{
N. Kawai ${ }^{1}$, M. Matsuoka ${ }^{1}$, A. Yoshida ${ }^{1}$, Y. Shirasaki ${ }^{1}$, M. Namiki ${ }^{1}$, K. Takagishi ${ }^{2}$, M. Yamauchi ${ }^{2}$, I. Hatsukade $^{2}$, \\ E.E. Fenimore ${ }^{3}$, and M. Galassi ${ }^{3}$ \\ 1 RIKEN, The Institute of Physical and Chemical Research, 2-1, Hirosawa, Wako, Saitama 351-0198, Japan \\ e-mail: nkawai@postman.riken.go.jp \\ 2 Miyazaki University, Faculty of Engineering, Gakuen-kihanadai-nishi, Miyazaki 880-2155, Japan \\ ${ }^{3}$ Los Alamos National Laboratory, MS D436, Los Alamos NM87545, U.S.A.
}

Received January 21; accepted March 10, 1999

\begin{abstract}
The Wide-field X-ray Monitor on HETE-2 consists of four Xe-filled 1-D position-sensitive proportional counters. It is sensitive to $\mathrm{X}$-rays between $2 \mathrm{keV}$ and $25 \mathrm{keV}$, and is designed to localize gamma-ray bursts in real time with $\sim 10^{\prime}$ accuracy. The design and basic characteristics of the detectors are presented.
\end{abstract}

Key words: instrumentation: detectors - gamma-rays: bursts - X-rays: general

\section{Introduction}

The HETE-2 mission is designed to determine many gamma-ray burst (GRB) positions at high accuracy $\left(10^{\prime}\right.$ for WXM, $10^{\prime \prime}$ for SXC) and broadcast them rapidly (within 10 seconds) to the follow-up observers. The HETE-2 satellite has three scientific instruments: the Wide-field X-ray Monitor (WXM), Soft X-ray Camera (SXC) and French Gamma Telescope (FREGATE). The SXC is a 1-D coded mask system using MIT-LL CCID-20's as the detecting elements, and has energy coverage of $500 \mathrm{eV}$ to $14 \mathrm{keV}$ with $2 \%$ resolution at $6 \mathrm{keV}$ and position resolution of $10^{\prime \prime}$ for bright bursts (1 Crab; $10 \mathrm{~s}$ ). The WXM has larger effective area and thus is more sensitive to weaker bursts than SXC, whereas $\mathrm{SXC}$ has superior spatial resolution. Thus the two X-ray instruments play an complemental role each other.

\section{Wide-field X-ray Monitor (WXM)}

The WXM consists of two identical units of onedimensional position sensitive X-ray detectors (Fig. 1). They are placed in orthogonal directions to each other for measuring the $X$ and $Y$ direction independently. One

Send offprint requests to: N. Kawai unit consists of a one-dimensional coded mask and two 1-D position-sensitive proportional counters (PSPCs) placed $187 \mathrm{~mm}$ below the mask. The coded mask is made of a plate of aluminum (0.5 mm thickness) and gold $(0.025 \mathrm{~mm})$ with a series of slits of randomly varying width (random mask). The location of the GRB is determined by measuring a set of two shift distances of the mask pattern in the $X$ and $Y$ direction. The mask pattern of WXM is selected from 100000 random patterns with the same open fraction and element size based on localization accuracy for GRBs.

Each PSPC has three carbon fiber anode wires with $10 \mu \mathrm{m}$ diameter in its upper cells and four tungsten wires in its lower cells, and filled with $1.4 \mathrm{~atm}$ Xenon gas with $3 \% \mathrm{CO}_{2}$ as quenching gas. The three upper cells are used for X-ray detection. They have a depth of $25.5 \mathrm{~mm}$ and a width of $\sim 27 \mathrm{~mm}$, and are separated by the cathode wires placed at intervals of $3.4 \mathrm{~mm}$. The four lower cells are used for rejecting charged particle events by anticoincidence, and have a depth of $11.5 \mathrm{~mm}$. The detection area of $120 \times 83.5 \mathrm{~mm}^{2}$ at the top side is sealed by $100 \mu \mathrm{m}$ thick Be windows.

The position of an incoming X-ray is determined by the charge-division method. That is, $L /(L+R)$ has a linear relation with the incident position, and $L+R$ measures photon energy. Here, $L$ and $R$ represent pulse height values measured at left and right ends respectively.

The proportional counters and electronics are provided by RIKEN and Miyazaki University, while the coded aperture and on-board localization software is provided by Los Alamos National Laboratory.

\section{Performance of PSPCs}

The performance and characteristics of the flight PSPCs were measured extensively. Here we present some results on their energy and position response measured with $8 \mathrm{keV}$ $\mathrm{X}$-rays collimated to $150 \mu \mathrm{m}$ radius at an applied anode 


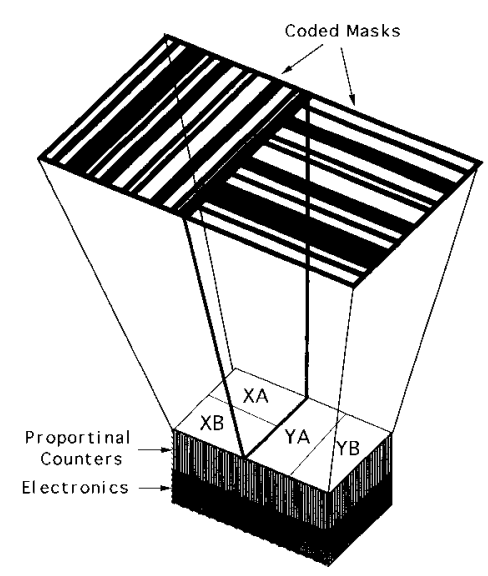

Fig. 1. Schematic view of the WXM: A set of $X \mathrm{~A}+X \mathrm{~B}$ $(Y \mathrm{~A}+Y \mathrm{~B})$ counters and a coded mask determine $\theta_{x}\left(\theta_{y}\right)$ that is a zenith angle of the GRB direction on the plane perpendicular to the corresponding mask pattern

voltage of $1700 \mathrm{~V}$. The collimated X-rays were projected on $87 \times 120$ grid points at $0.94 \mathrm{~mm}$ intervals on PSPC aperture. It has been noticed at the previous calibration of WXM/HETE-1 (Yamauchi et al. 1997; Tokanai 1997) that the self-induced space charge effect degrades energy resolution and its linearity at high anode voltages. In the HETE-2 counters, the energy resolution becomes worse at $\geq 1600 \mathrm{~V}$ while the position resolution gradually improves at higher voltages and reaches $0.6 \mathrm{~mm}$ (FWHM) around $1700 \mathrm{~V}$. Therefore we choose to operate around $1650 \mathrm{~V}$ in order to achieve good position resolution of $<1 \mathrm{~mm}$, which is required for PSPC/WXM to localize GRBs with $10^{\prime}$ accuracy in 2-second integration time. Here we present some results from a counter at $1700 \mathrm{~V}$ anode voltage.

The linearity of the output pulse height (sum of the charges collected at the left and right ends) to the incidence photon energy was measured for one PSPC using monochromatic X-ray beams with energies $6-24 \mathrm{keV}$ with $2 \mathrm{keV}$ step. While at the applied voltage of $1400 \mathrm{~V}$ there is a good linearity between them, at $1700 \mathrm{~V}$ the linearity is not conserved due to the self-induced space charge effect. The energy resolution is mostly uniform $(22 \%$ at $8.04 \mathrm{keV}$ with $1700 \mathrm{~V}$ anode voltage) except near the wire ends $( \pm 60 \mathrm{~mm})$ where the electric field is distorted by the wall of the counter.

The position of an incident X-ray is determined from $X=L /(L+R)$, the ratio of the charge collected at the left and right ends of the anode wire. Ideally the ratio $X$ is expected to have a linear relation with the incident position $x$ along the anode. In reality there is nonlinearity near the ends of anode wire (Fig. 2). We use an empirical analytical formula to calculate the incident position $x_{\text {cal }}$ from the measured charge ratio $X$. The parameters of the formula are determined for each anode wire by fitting the measured curve. The position resolution was also found to be mostly uniform at $0.6-0.7 \mathrm{~mm}$ except near the

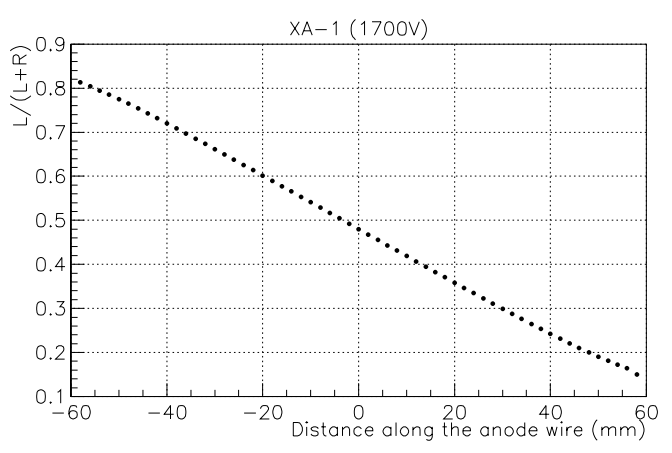

Fig. 2. The relation between pulse height ratio $L /(L+R)$ and incident position of X-rays

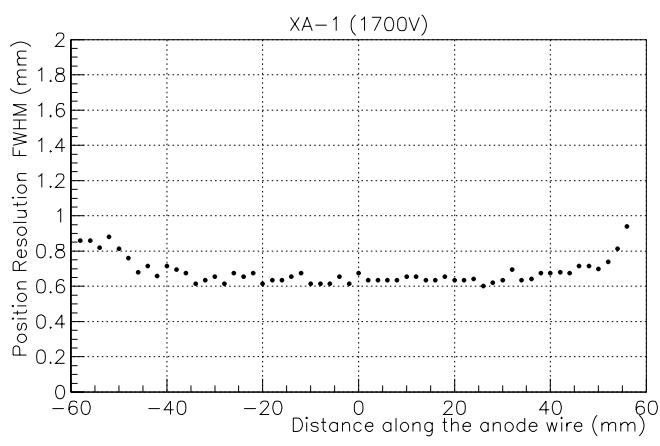

Fig. 3. Position resolution (FWHM; mm) as a function of incident position along the anode wire

wire ends. In Fig. 3 the position resolution expressed by the FWHM of the distribution of $x_{\text {cal }}$ is plotted against incident position $x$.

\section{Expected WXM sensitivity}

We have carried out Monte Carlo simulation to estimate the sensitivity of WXM to GRBs. Here, the photon energy was limited within $3-10 \mathrm{keV}$ to reduce the blurring due to penetration. Then the position histogram in $0.5 \mathrm{~mm}$ bin was simulated for the detector geometry. The GRB direction was reconstructed by cross-correlating the simulated data with the number of templates corresponding to various incidence angles. In the simple simulation with 2 second integrations, the correct position was obtained for half of the events that occur within the field of view of the WXM with peak fluxes of $4 \mathrm{ph} / \mathrm{cm}^{2} / \mathrm{s}\left(410^{-8} \mathrm{erg} / \mathrm{cm}^{2} / \mathrm{s}\right.$ over the $3-10 \mathrm{keV}$ range) and, under favorable conditions, some events down to $0.4 \mathrm{ph} / \mathrm{cm}^{2} / \mathrm{s}$. Assuming the BATSE $\log N-\log P$ relation, this sensitivity results in localization of about 30 GRBs per year.

\section{References}

Yamauchi M., et al., 1997, in All-Sky X-Ray Observations in the Next Decade, Matsuoka M. and Kawai N. (eds.), pp. 297-302

Tokanai F., 1997, Ph.D thesis, Saitama University 